\title{
Absorption Bands of Carbon Dioxide* From 5.3 to 4.6 Microns
}

\author{
Arthur G. Maki, Earle K. Plyler, and Robert J. Thibault
}

(February 12, 1963)

\begin{abstract}
Measurements have been made of the frequencies of the infrared absorption lines of $\mathrm{CO}_{2}$ in the region from $1850 \mathrm{~cm}^{-1}$ to $2150 \mathrm{~cm}^{-1}$. Observations were made at various pressures and pathlengths up to a maximum of 72 meter-atmospheres. Vibration-rotation constants were obtained characterizing the transitions $11^{1 c} 0-000,11^{1 d} 0-000,03^{1 c} 0-000,03^{1} d 0-000,200-01^{1 c} 0$, $12^{2 c 0}-01^{1 c} 0,12^{2 d} 0-01^{1 a} 0$ for $\mathrm{C}^{12} \mathrm{O}_{2}$. The $11^{1 c} 0-000$ band due to the $\mathrm{C}^{13} \mathrm{O}_{2}$ molecule was also measured.
\end{abstract}

\section{Introduction}

The infrared spectrum of $\mathrm{CO}_{2}$ has been exhaustively studied by Courtoy who has compiled a critical survey of the energy levels and vibrationrotation constants for the various isotopic species of that molecule $[1,2]{ }^{1}$

The five micron region contains two weak absorption bands due to the transitions $03{ }^{1} 0-000$ and $11^{1} 0-000$. These two bands form a Fermi resonant diad and are rather important for determining the best potential constants for $\mathrm{CO}_{2}$. Migeotte, Neven, Swenson, and Benedict have reported previous measurements on this region [3], but the resolution and wavelength accuracy available at that time was not quite as good as that now available in this laboratory. Because of slight disagreements between calculated and observed values, it was felt that those measurements should be repeated. In addition we have been able to resolve and analyze two $Q$ branches for which the constants seem not to have been previously reported. The intensities of these bands are of great interest and of particular interest is the intensity anomaly which causes the $P$ branch to be strong while the $R$ branch is weak. Since this anomaly has already been discussed by Benedict in quantitative terms [4], it will not be further discussed here.

*This research was supported in part by the U.S. Air Force Geophysies Research Directorate, Bedford, Mass.

1 Figures in brackets indicate the literature references at the end of this paper.

\section{Experimental Procedure}

The spectra were measured on the NBS high resolution infrared spectrometer described elsewhere [5]. Measurements were made using one of two gratings and a liquid nitrogen cooled PbSe detector. The highest resolution was obtained by using a 1,860 lines/in. grating double passed in third order. With this grating measurements were interpolated from a wavelength scale provided by measurements on a 7,500 lines/in. grating. Only lines that were not resolved with the finer grating were measured with the 1,860 lines/in. grating. Most of the measurements reported here were obtained directly from measurements with a 7,500 lines/in. grating used in the first order and double passed. Calibration with this grating was carried out by means of accurately measured rare gas spectra with a Fabry-Perot interferometer fringe system superimposed on the spectra for interpolation as described in reference 6 .

The majority of the measurements were made with a pathlength of $24 \mathrm{~m}$ and pressures up to $1 \mathrm{~atm}$. Pressures up to $3 \mathrm{~atm}$ were used in trying to observe lines due to the weaker transitions, but the increased pressure broadening of the lines usually resulted in a reduced sensitivity to the weaker lines. To achieve the highest resolution of the strong $Q$ branches a $4 \mathrm{~m}$ path was used thus decreasing reflection losses in the absorption cell. 


\section{Analysis and Results}

\subsection{Analysis}

Except for the $Q$ branches the spectra were all analyzed using a least squares computer program to fit the observed frequencies to the power series

$$
\begin{aligned}
\nu_{\mathrm{obs}}=\nu_{0}+\left(B^{\prime}+B^{\prime \prime}\right) m+ & \left(B^{\prime}-B^{\prime \prime}\right) m^{2} \\
& -2\left(D^{\prime}+D^{\prime \prime}\right) m^{3}-\left(D^{\prime}-D^{\prime \prime}\right) m^{4}
\end{aligned}
$$

where the symbols have their usual meaning. The $Q$ branch lines were fitted to the equation

$$
\nu_{\mathrm{obs}}=\nu_{0}+\left(B^{\prime}--B^{\prime \prime}\right) J(J+1)-\left(D^{\prime}-D^{\prime \prime}\right) J^{2}(J+1)^{2} .
$$

The calculated and observed constants are tabulated in table 1 . Where no observed constants are given, the data were insufficient to obtain those constants and the calculated values were used in the band analysis. Since it was felt that Courtoy's values for $B^{\prime \prime}$ and $D^{\prime \prime}$ were more accurate than values obtainable from these measurements, Courtoy's values, as given in reference 2 , were used to determine the other constants for all the bands reported here.

The calculated values of $B^{\prime}$ are those given by Courtoy [2] with the incorporation of a correction term due to the Coriolis interaction discovered by Courtoy and explained by Andrade e Silva and Amat [7]. The correction to the $B^{\prime}$ terms was made in the manner described by Courtoy and Triaille [8]. This correction was quite small for these energy levels, the largest correction being about $-1.4 \times 10^{-5} \mathrm{~cm}^{-1}$ for $B_{12^{2} 0^{2}}$.

The equations given by Nielsen, Amat, and Goldsmith $[9,10]$ were used to determine the approximate $l$-type resonance and Fermi resonance corrections necessary for the centrifugal distortion terms $\left(D_{v}\right)$. No attempt was made to include the effect of an additional $J(J+1)$ dependence in the Fermi resonance matrix element which has recently been predicted by Maes [11]. Since the calculated values of $\Delta D$ are probably as good as the measured values, the calculated values of $\Delta D$ were used in determining the final values of $\Delta B$. In those cases where a value of $\Delta D$ was experimentally determinable, both observed and calculated $\Delta D$ 's gave nearly the same $\Delta B$ 's.

In table 1 the standard deviations are given for the various constants. These deviations were determined solely by the scatter of the data and do not reflect possible absolute errors.

Table 2 and 3 list the observed and calculated frequencies (wavenumbers) for the absorption lines in the various bands. Where comparison is possible, the observed wavenumbers given in table 2 and 3 generally agree with those listed in reference 3 to within a tenth of a wavenumber $\left(\mathrm{cm}^{-1}\right)$. For the least squares computations line measurements to three decimal places were used. Because of the doubtful significance of the third decimal place, the wavenumbers given in tables 2 and 3 have been rounded off to two decimal places.

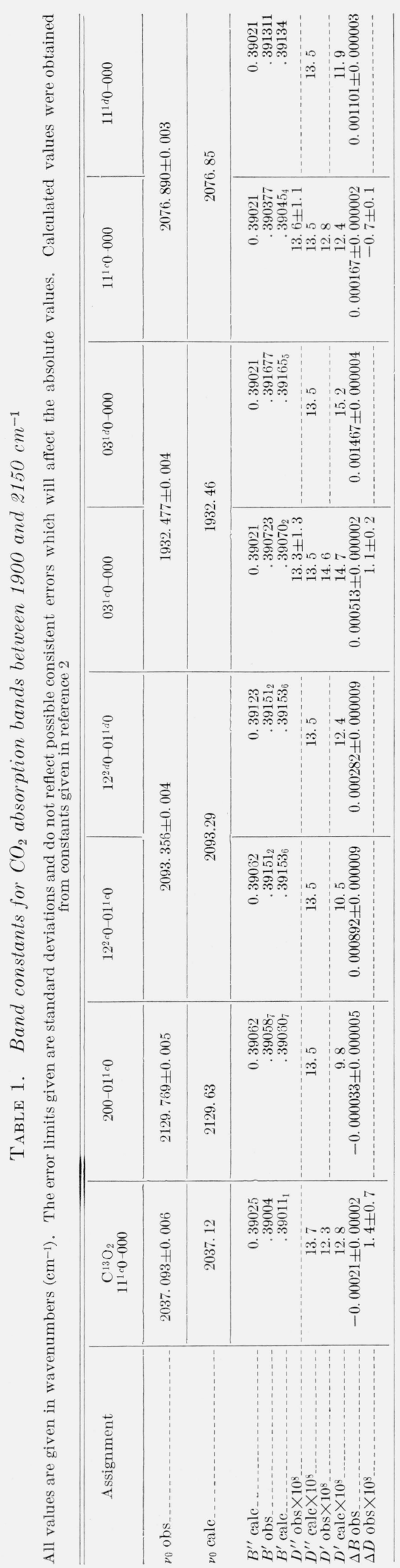


TABLE 2. Wavenumbers $\left(\mathrm{cm}^{-1}\right)$ of weak absorption bands due to $\mathrm{C}^{13} \mathrm{O}_{2}$ and $\mathrm{C}^{12} \mathrm{O}_{2}$ in 5 micron wavelength region

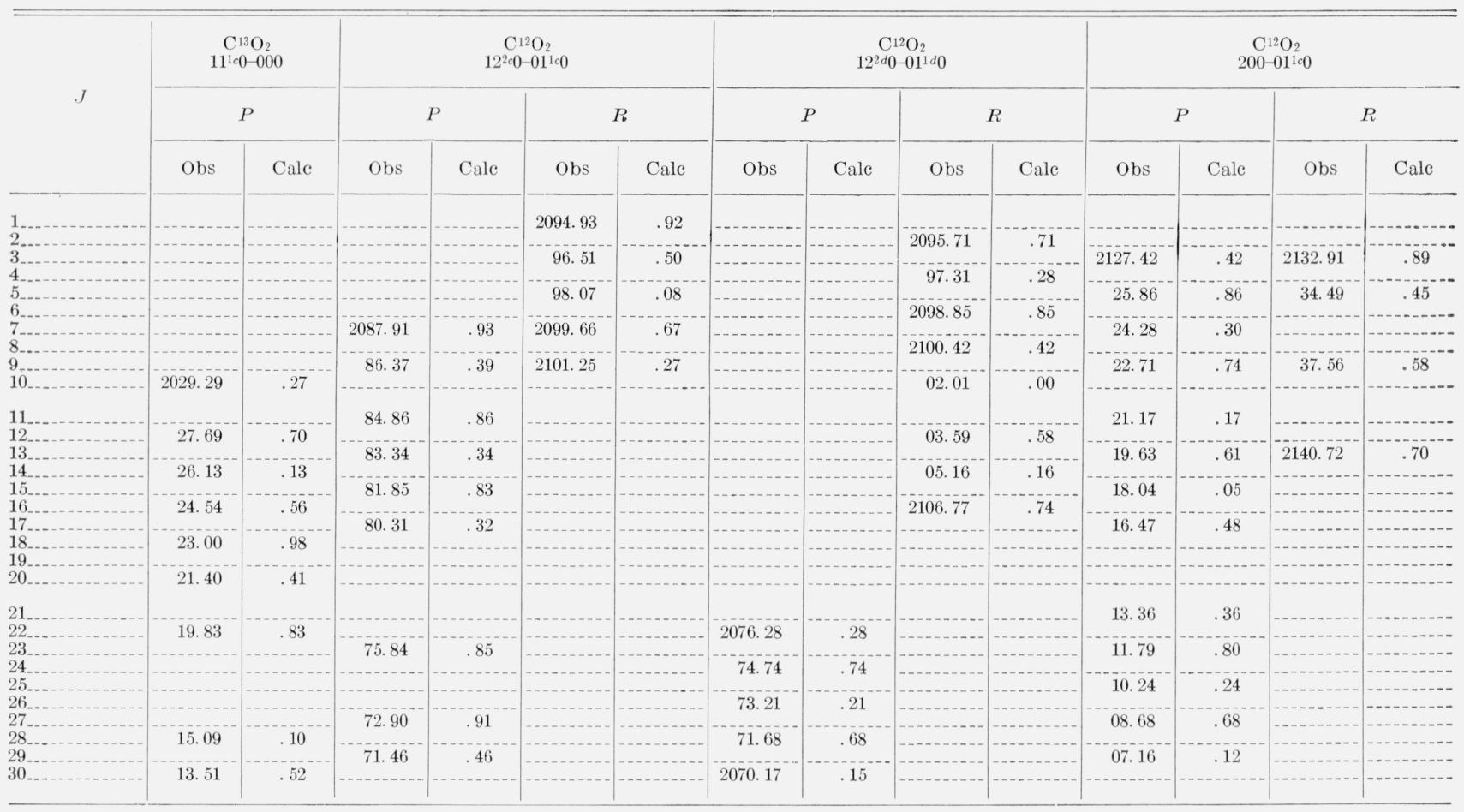

\section{2. $\mathrm{C}^{13} \mathrm{O}_{2}$ Band at $2037 \mathrm{~cm}^{-1}$}

The band due to the $11^{1 c} 0-000$ transition of $\mathrm{C}^{13} \mathrm{O}_{2}$ was measured at $2037.09 \mathrm{~cm}^{-1}$. This band is part of a Fermi doublet of which the other band lies at about $1896.54 \mathrm{~cm}^{-1}$. The other band was not detected in this work even at the highest pressures and pathlengths used. It is expected to be considerably weaker than the $11^{1} 0-000$ band due in part to the diminished Fermi resonance in $\mathrm{C}^{13} \mathrm{O}_{2}$. Consequently, it will probably be necessary to use isotopicly enriched samples before this $03^{1} 0-000$ transition will be measurable. The $Q$ branch for the $11^{1} 0$ band of $\mathrm{C}^{13} \mathrm{O}_{2}$ was not resolved in this work. This is understandable since the $\Delta B$ term, which governs the $Q$ branch line spacing, is predicted to be rather small and relatively large pressures were necessary to observe this weak band. Furthermore, this $Q$ branch is overlapped by the $P$ branch of the much stronger $\mathrm{C}^{12} \mathrm{O}_{2}$ band.

Table 1 gives a comparison of the calculated and observed values of the various vibration-rotation constants necessary to describe this band. The discrepancies in $\nu_{0}$ and $B^{\prime}$ are believed to be greater than the experimental error and are consequently indicative of either a very small error in Courtoy's constants or the neglect of some terms in the potential expansion. Such errors are not unexpected for $\mathrm{C}^{13} \mathrm{O}_{2}$ as Courtoy did not have a surfeit of data from which to derive his constants. Table 2 lists the lines measured and the values calculated using the constants given in table 1 .

\section{3. $\mathrm{CO}_{2}$ Bands Between 1900 and $2150 \mathrm{~cm}^{-1}$}

The strongest $\mathrm{CO}_{2}$ absorption bands in the $5 \mu$ region are those due to the Fermi doublet $03^{1} 0-000$ and $11^{1} 0-000$. For these transitions a rather large number of lines have been measured in the $P$ and $R$ branches and the $Q$ branches were sufficiently well resolved to yield good values for $\Delta B$.

Two more bands due to the transitions $200-01^{1} 0$ and $12^{2} 0-01^{1} 0$ have been measured in this region. The latter band is split into $c$ and $d$ components. When treated individually the two components vield values $B_{12}{ }^{2}$ which should be equal and in fact are found to be the same to within $5 \times 10^{-6} \mathrm{~cm}^{-1}$. Such agreement indicates that the standard deviations quoted are realistic. The values reported in table 1 for the $12^{2} 0-01^{1} 0$ bands were obtained by assuming that the $c$ and $d$ components of the upper vibrational level had the same vibrational energy (band center) and $B_{v}$ values.

Similarly the analysis of the $c$ and $d$ components of each of the two Fermi doublet bands $3 \nu_{2}^{1}$ and $\nu_{1}+\nu_{2}^{1}$ was carried out simultaneously so as to yield the the same values for the band centers of the $c$ and $d$ components.

The important constants $\nu_{0}$ and $\Delta B$ for the Fermi doublet are in slight disagreement with the values predicted by Courtoy's constants. At least in the case of $\nu_{1}+\nu_{2}$ this discrepancy is greater than experimental error. The band centers for the other bands in this region are in even greater disagreement with the calculated values. 
TABLE 3. Strongest $\mathrm{CO}_{2}$ absorption bands between 4.6 and 5.3 microns

Line measurements are given in units of wavenumbers $\left(\mathrm{cm}^{-1}\right)$

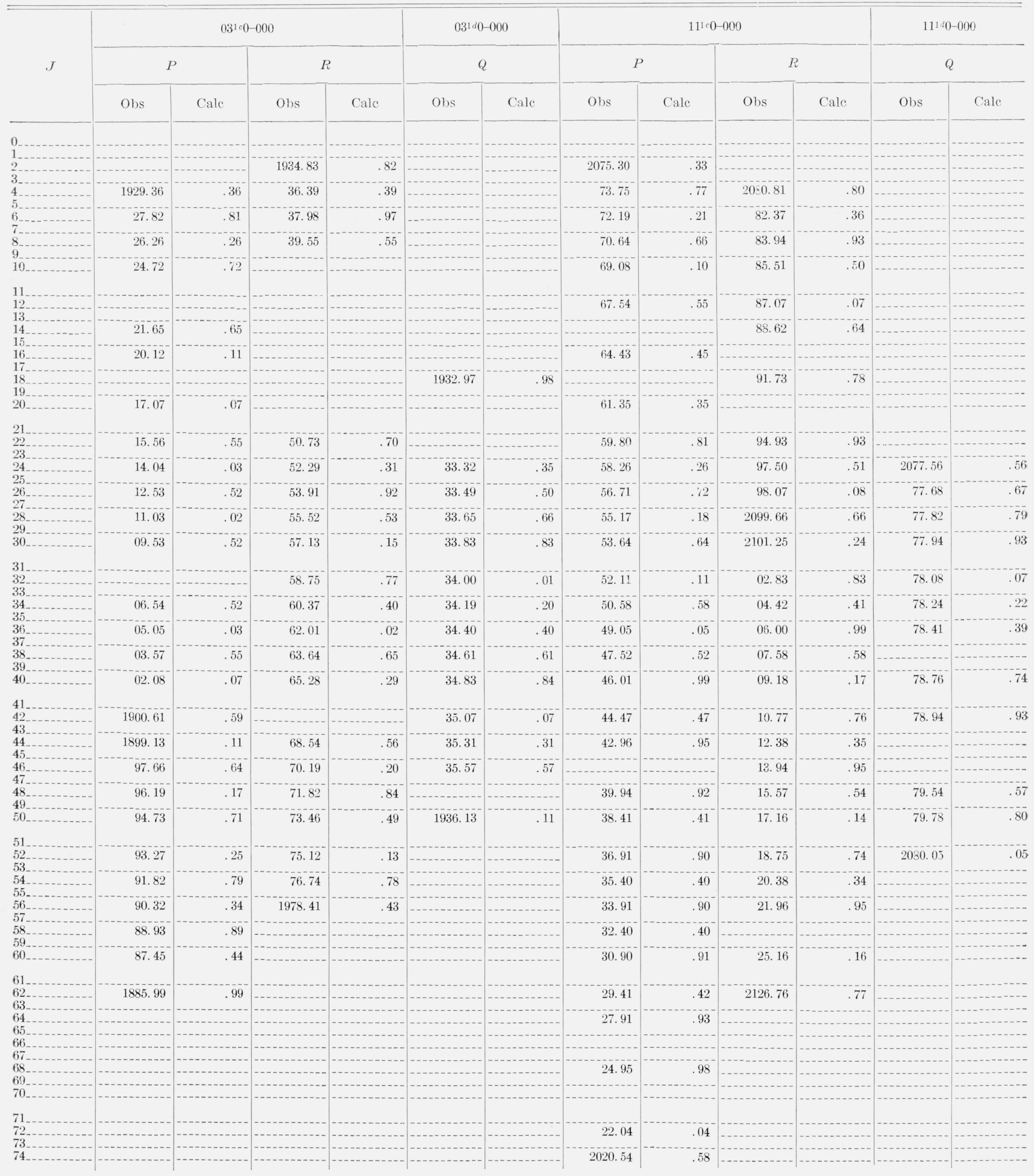


No attempt has been made to reevaluate the various vibration-rotation constants in order to obtain a better fit with the data presented here. Such a task would be a major undertaking and Courtoy has indicated [8] that such work is in progress elsewhere. The fact that both $c$ and $d$ components of the two resonating diads have been resolved does allow one to make a direct evaluation of some combinations of constants.

For a resonance diad the sum of the $l$ doubling terms $\left(q_{v}\right)$ for the two resonating energy levels is constant and independent of the degree of resonance. From table 1 one finds $q_{11{ }^{1}{ }_{0}}+q_{03^{1}{ }_{0}}=188.8 \times 10^{-5}$ $\mathrm{cm}^{-1}$. Correcting for the interaction between the $c$ levels and $\nu_{3}$ this becomes $187.9 \times 10^{-5} \mathrm{~cm}^{-1}$.

Since

$$
q_{v}=\frac{1}{2}\left(v_{2}+1\right)\left[q_{0}-q_{1} v_{1}-q_{2} v_{2}-q_{3} v_{3}\right]
$$

and

$$
q_{01^{1} 0}=61 \times 10^{-5}=q_{0}-q_{2}
$$

then

$$
q_{11}{ }^{1}{ }_{0}+q_{03^{1}{ }_{0}}=183 \times 10^{-5}-4 q_{2}-q_{1} .
$$

This gives $4 q_{2}+q_{1}=-4.9 \times 10^{-5} \mathrm{~cm}^{-1}$. Similarly the sum of $\Delta B_{11^{1}{ }^{0-000}}$ and $\Delta B_{03}{ }^{1}{ }^{-0}-000$ yields $-\alpha_{1}-4 \alpha_{2}+$ $\gamma_{11}+10 \gamma_{22}+\gamma_{12}+2 \gamma_{l l}=162.9 \times 10^{-5} \mathrm{~cm}^{-1}$ when the Coriolis resonance correction is made. This compares very favorably with the value of $164.5 \times 10^{-5} \mathrm{~cm}^{-1}$ calculated using the constants given on page 128 of reference 2. It is difficult to tell whether the difference of $1.6 \times 10^{-5} \mathrm{~cm}^{-1}$ is significant.

The rather large deviations from the predicted positions of some of the band centers which has been found in this work and that of Plyler, Tidwell, and Benedict [12] may be due, at least in part, to the neglect of the constants $x_{17 l}, x_{2 l l}$, and $x_{3 l l}$ in the vibrational energy expansion. These constants are necessary for $\mathrm{N}_{2} \mathrm{O}$ [13] and have recently been found to be important for the vibrational analysis of HCN [14] and DCN.
The authors acknowledge the assistance of Joseph Hilsenrath in using the least squares computer program, and to Dr. W. S. Benedict for his suggestions concerning these measurements.

\section{References}

[1] C. P. Courtoy, Can. J. Phys. 35, 608 (1957).

[2] C. P. Courtoy, Ann. Soc. Sci. Bruxelles, Ser. I, 73, 5 (1959).

[3] M. Migeotte, L. Neven, J. Swensson, and W. S. Benedict, Mem. Soc. Roy. Sci. Liege, Special Vol. 2 (1957).

[4] W. S. Benedict, APCRC-TR-56-275 (1956).

[5] E. K. Plyler and L. R. Blaine, J. Research NBS 62, 7 (1959) RP2922.

[6] E. K. Plyler, L. R. Blaine, and E. D. Tidwell, J. Research NBS 55, 279 (1955) RP2630.

[7] Andrade e Silva and G. Amat, J. Mol. Spect. 9, 354 (1962).

[8] C. P. Courtoy and E. A. Triaille, J. Mol. Spect. 9, 339 (1962).

[9] G. Amat, M. Goldsmith, and H. H. Nielsen, J. Chem. Phys. 24, 44 (1956).

[10] H. H. Nielsen, G. Amat, and M. Goldsmith, J. Chem. Phys. 26, 1060 (1957).

[11] S. Maes, J. Mol. Spect. 9, 204 (1962).

[12] E. K. Plyler, E. D. Tidwell, and W. S. Benedict, J. Opt. Soc. Am. 5\%, 1017 (1962).

[13] E. D. Tidwell, E. K. Plyler, and W. S. Benedict, J. Opt. Soc. Am. 50, 1243 (1960).

[14] A. G. Maki and L. R. Blaine, J. Mol. Spect. (in press). 